\title{
White adipose tissue browning: A novel and effective therapeutic strategy and approach for type 2 diabetes
}

\author{
Guosheng $\mathrm{LI}^{1 *}$, Xuhan $\mathrm{LIU}^{2}$, Jinglei $\mathrm{MU}^{2}$, Qingyan $\mathrm{XU}^{2}$, Qingmin $\mathrm{MENG}^{2}$, Lizhi ZHANG ${ }^{1}, \mathrm{Lu}_{\mathrm{FENG}}{ }^{1}$, Chang SHI ${ }^{1}$, Pengxin ZHANG ${ }^{1}$ and \\ Huali WANG ${ }^{1}$ \\ ${ }^{1}$ Department of Pathology, the First Affiliated Hospital of Dalian Medical University; 222 Zhong Shan Road, Dalian 116011, China \\ ${ }^{2}$ Department of Endocrinology, Dalian Municipal Central Hospital; 42 Xue Gong Road, Dalian 116003, China
}

\begin{abstract}
The global epidemic of metabolic diseases such as obesity and type 2 diabetes continues to raise and needs novel and more effective therapeutic strategies and approaches. Obesity is ultimately induced by excess and ectopic fat accumulation and is a major risk factor for type 2 diabetes. Excess fat accumulation in white adipose tissues, linking to adipose tissue dysfunction, causes other insulin-responsive organs ectopic fat accumulation and insulin resistance and then leads to obesityrelated type 2 diabetes. So adipose tissues and its molecular biology of formation and function have heightened scientific interest. Adipose tissues, a large, heterogenic, pleiotropic and rather complex metabolic organ, play a central role in controlling and regulating glucose homeostasis and systemic energy metabolism. The adipose tissue organ is distributed at distinct anatomical depots and there are three different classes of adipocytes; white, brown and beige adipocytes. Among these, Beige adipocytes are induced to develop in white adipose tissue by white adipose tissue browning to produce heat. Beige adipocytes possess intermediate characteristics of both white adipocytes and brown adipocytes and can be induced by pharmacological substances. Excess energy accumulation in white adipose tissue could be reduced the activation of brown adipose tissue and the browning in white adipose tissues. Thus, activation of brown adipose tissue/beige in white adipose tissue mediated thermogenic activity will counteract insulin resistance and type 2 diabetes. Beige adipocytes are potential targets for new attractive therapeutic strategy of obesity and type 2 diabetes.
\end{abstract}

\section{Introduction}

The occurrences of obesity continue to rise worldwide [1]. Obesity is the result of a long-term imbalance between energy intake and energy expenditure. Excess fat accumulation in excess or expansion of white adipose tissues, a kind of disturbance of storing excess energy, in obesity will cause other insulin-responsive organs such as the liver, skeletal muscle, and pancreas ectopic fat accumulation and insulin resistance, which can lead to obesity-related type 2 diabetes [2]. Humans possess three different types of adipocytes (white, brown and beige adipocytes) and two different types of adipose depots: subcutaneous adipose tissue and visceral adipose tissue. The phenomenon that beige adipocyte differentiation within white adipose tissue, referred to as browning, involves the conversion of non-classic brown adipocytes to beige/ brown-like adipose tissue bearing thermogenic, fat-burning properties, and this phenomenon has slowed the progression of obesity-related type 2 diabetes [3]. Thus, browning of white adipose tissue is seen as a possible mechanism for potential anti diabetes effects. The molecular regulation underlying the thermogenic browning process has not been entirely elucidated. The investigation of browning of white adipose tissue has recently emerged as an attractive therapeutic strategy for obesity and type 2 diabetes.

\section{The relationship between obesity and type 2 diabetes}

Excess and ectopic fat accumulation in obesity, a serious and major public health problem worldwide, is driven by the disruption and disequilibrium in energy balance and is a major risk factor for developing the onset of type 2 diabetes [4,5]. Obesity and type 2 diabetes associated with a variety of comorbidities cause a substantial economic burden for society and contribute to mortality around the world [6]. Excess fat accumulation in white adipose tissues (lipid/nutrient overload) in obesity causes adipose depots/tissues dysfunction, other insulin-responsive organs ectopic fat accumulation and then insulin resistance and type 2 diabetes ensue [2].

\section{The characters of adipose tissue in obesity}

An excess of white adipose tissue accumulation, usually visceral white adipose tissue, is an important character of obesity and tightly associated with the disturbance of storing excess energy and the inflammatory response in the adipocytes $[7,8]$. An excess or expansion of white adipose tissue comes from both the hypertrophy of existing adipocytes and hyperplasia of adipocyte pre-cursors [1]. Excess fat accumulation in excess or expansion of white adipose tissues in obesity impairs subcutaneous adipose tissue resistance to its dysfunction, also causes other insulin-responsive organs ectopic fat accumulation, dysfunction and systematic insulin resistance, which finally lead to obesity-related type 2 diabetes [2]. In addition, Excess energy accumulation in white adipose tissues could be reduced the activation of brown adipose tissue and the browning in white adipose tissues [9].

*Correspondence to: Guosheng LI, Department of Pathology, The First Affiliated Hospital of Dalian Medical University, 222 Zhong Shan Road, Xang Gang District, Dalian, 116011, China, E-mail: guoshengli998@163.com

Key words: obesity, type 2 diabetes, browning of white adipose tissue, beige adipocyte

Received: October 24, 2018; Accepted: November 14, 2018; Published: November 19, 2018 


\section{The biological role of adipose tissue}

The rising global incidence of obesity and type 2 diabetes have increased the urgency in better understanding all aspects of cell biological processes of adipose tissue formation and function and necessitates research therapeutic interventions on adipose tissues [1012]. Adipose tissues, a large, heterogenic, pleiotropic and rather complex metabolic organ that is highly active and essential, play a central role in controlling and regulating glucose homeostasis and systemic energy metabolism and maintaining energy homeostasis by storing lipids, dissipating them as heat, and producing various adipokines. Function of adipose tissue changes according to the site of the adipose depot, the cell composition of the tissue and the energy status of the organism. The adipose tissue organ located all along the body is distributed at distinct anatomical depots and there are three different classes of adipocytes : white, brown and beige adipocytes which are interacted with extracellular stroma, immune, and neural cells [13].

\section{The classes of adipocytes and their functions}

These three different classes of adipocytes serve different functions. White adipocytes store excess nutrients/energy in the form of lipid (triglycerides) and control the lipid mobilization and distribution to supply the demand of energy imposed by physiological cues in other organs. White adipose tissue also serves as an important endocrine organ and produces bioactive factors (adipokines) to communicate with other organs and modulate metabolic pathways [14]. Brown adipocytes are responsible for combusting lipids and glucose to produce heat (thermogenesis) and dissipate energy and form discrete and relatively homogenous depots of brown adipose tissue. Beige adipocytes (brown cell-like "beige" adipocytes) are induced to develop in white adipose tissue depots through a process called white adipose tissue browning to produce heat on certain conditions [15]. These beige cells can be induced by cold and pharmacological substances, therefore, they are called "inducible brown adipocytes". Beige adipocytes possess intermediate characteristics of both white adipose tissue and brown adipose tissue. Brown and beige adipocytes expend chemical energy to produce heat through uncoupling oxidation from energy production and regulate body temperature and body weight [16].

\section{The classes of adipose tissue and their structural and functional characters}

There are two structurally and functionally different depots called subcutaneous adipose tissue and visceral adipose tissue. Subcutaneous adipose tissue showed some protective endocrine and inflammatory features. When subcutaneous adipose tissue is unable to expand sufficiently, fat overflows towards metabolically adverse ectopic depots. Subcutaneous adipose tissue is apt to "browning" and contributes to its favourable metabolic effects. Obesity, low physical activity and other environmental factors impair subcutaneous adipose tissue resistance to its dysfunction and promote development of metabolic disorders. Humans possess three different types of adipocytes and subcutaneous adipose tissue and visceral adipose tissue [17].

\section{The relationship between adipose tissue and obesity-related type 2 diabetes}

The coordinate action of the adipocytes/tissues is important to ensure an optimal metabolic status. However, when one or multiple of these adipose depots/tissues dysfunction result from lipid/nutrient overload, then insulin resistance and type 2 diabetes ensue. Activation of brown adipose tissue/beige in white adipose tissue mediated thermogenic activity counteracts insulin resistance, lipotoxic effects and metabolic diseases including type 2 diabetes. Thus, brown/brite adipocytes are potential targets to stimulate energy expenditure for new attractive therapeutic strategy of obesity and type 2 diabetes $[13,18]$.

\section{The induction of beige cells and mechanisms}

Excess energy accumulation in white adipose tissue could be reduced the activation of brown adipose tissue and the browning in white adipose tissue [9]. Browning of white adipose tissue has potential antidiabetic effects, yet the mechanisms remain to be fully understand [19]. The theory about the origin of beige adipocytes is de novo differentiation from resident progenitor cells and trans differentiation from mature adipocytes. The theory about de novo differentiation begins with the differentiation of progenitor cells to a beige preadipocyte phenotype. The trans differentiation theory proposes that mature cells are the source of beige adipocytes. Hormones, cytokines, nutrients and drugs able to modulate the route of the intracellular events involved in the recruitment process of beige precursors [9]. BMP7 and EBF2, which are critical factors for the commitment of progenitor cells to beige preadipocytes were triggered by adipogenic stimulation. Then the expression of characteristic thermogenic genes such as uncoupling protein (UCP)-1, PR domain containing 16 (PRDM16), and Zfp516 was activated by the transcriptional machinery of the browning program. A key step in the differentiation and function of browning is the deacetylation of peroxisome proliferator-activated receptor (PPAR) $\gamma$ by SIRT1 and the consequent mitochondrial biogenesis [20]. Browning program also involves the expression of many transcription factors, such as PGC-1a and C/EBPs. Importantly, the expression of PRDM16 and its binding partner $\mathrm{C} / \mathrm{EBP} \beta$ is sufficient to promote differentiation into functional brown fat cells [21].

\section{Conclusion}

Adipose tissues distributed at distinct anatomical depots (e.g. subcutaneous adipose tissue vs. visceral adipose tissue) and there are three different classes of adipocytes: white, brown and beige adipocytes, which are both metabolic health determinants with therapeutic implications. Induction of browning of white adipose tissue is an attractive target therapy for the prevention of type 2 diabetes. Browning of white adipose tissue can improve glucose and lipids homeostasis and may be a means of manipulating systemic insulin resistance. Future work will further target the manipulation of thermogenic adipocytes by pharmacological and environmental means, which will greatly enhance our understanding of beige adipocyte biology.

\section{Funding}

This work was supported by Natural Science Foundation of Liaoning Province of China (20170540260)

\section{Conflicts of interest}

Authors state no conflicts of interest.

\section{Acknowledgement}

Guosheng Li is funded by Natural Science Foundation of Liaoning Province of China (20170540260).

\section{References}

1. Aldiss P, Betts J, Sale C, Pope M, Budge H, et al. (2018) Exercise-induced 'browning' of adipose tissues. Metabolism 81: 63-70. [Crossref]

2. Goto T, Hirata M, Aoki Y (2017) The hepatokine FGF21 is crucial for peroxisome proliferator-activated receptor- $\alpha$ agonist-induced amelioration of metabolic disorders in obese mice. J Biol Chem 292: 9175-9190. [Crossref] 
3. Tsiloulis T, Carey AL, Bayliss J (2018) No evidence of white adipocyte browning after endurance exercise training in obese men. Int J Obes (Lond) 42: 721-727. [Crossref]

4. Matsukawa T, Villareal MO, Motojima H, Isoda H (2017) Increasing cAMP levels of preadipocytes by cyanidin-3-glucoside treatment induces the formation of beige phenotypes in 3T3-L1 adipocytes. J Nutr Biochem 40: 77-85. [Crossref]

5. Schlein C, Heeren J (2016) Implications of thermogenic adipose tissues for metabolic health. Best Pract Res Clin Endocrinol Metab 30: 487-496. [Crossref]

6. Kong X, Williams KW, Liu T (2017) Genetic mouse models: the powerful tools to study fat tissues. Methods Mol Biol 1566: 99-107. [Crossref]

7. Gomez-Hernandez A, Beneit N, Diaz-Castroverde S, Escribano O (2016) Differential role of adipose tissues in obesity and related metabolic and vascular complications. Int $J$ Endocrinol 2016: 1216783. [Crossref]

8. Vargas-Castillo A, Fuentes-Romero R, Rodriguez-Lopez LA, Torres N, Tovar AR (2017) Understanding the biology of thermogenic fat: is browning a new approach to the treatment of obesity? Arch Med Res 48: 401-413. [Crossref]

9. Forest C, Joffin N, Jaubert AM, Noirez P (2016) What induces watts in WAT? Adipocyte 5: 136-152. [Crossref]

10. Imran KM, Yoon D, Kim YS (2018) A pivotal role of AMPK signaling in medicarpinmediated formation of brown and beige adipocytes from C3H10T1/2 mesenchymal stem cells. Biofactors 44: 168-179. [Crossref]

11. Steinbring J, Graja A, Jank AM (2017) Flow cytometric isolation and differentiation of adipogenic progenitor cells into brown and brite/beige adipocytes. Methods Mol Biol 1566: 25-36. [Crossref]

12. Hepler C, Gupta RK (2017) The expanding problem of adipose depot remodeling and postnatal adipocyte progenitor recruitment. Mol Cell Endocrinol 445: 95-108. [Crossref]
13. Carobbio S, Pellegrinelli V, Vidal-Puig A (2017) Adipose tissue function and expandability as determinants of lipotoxicity and the metabolic syndrome. Adv Exp Med Biol 960: 161-196. [Crossref]

14. Luo L, Liu M (2016) Adipose tissue in control of metabolism. J Endocrinol 231: R77-R99. [Crossref]

15. Oishi Y, Manabe I (2017) Adipose stem cell system. Clin Calcium 27: 795-801. [Crossref]

16. Chen Y, Pan R, Pfeifer A (2016) Fat tissues, the brite and the dark sides. Pflugers Arch 468: 1803-1807. [Crossref]

17. Mao L, Nie B, Nie T, Hui X, Gao X, et al. (2017) Visualization and quantification of browning using a ucp1-2a-luciferase knock-in mouse model. Diabetes 66: 407-417. [Crossref]

18. Pandzic Jaksic V, Grizelj D (2016) Under the surface of subcutaneous adipose tissue biology. Acta Dermatovenerol Croat 24: 250-260. [Crossref]

19. Meng W, Liang X, Chen H (2017) Rheb inhibits beiging of white adipose tissue via PDE4D5-Dependent downregulation of the cAMP-PKA signaling pathway. Diabetes 66: 1198-1213. [Crossref]

20. Abdul-Rahman O, Kristof E, Doan-Xuan QM (2016) AMP-Activated Kinase (AMPK) activation by aicar in human white adipocytes derived from pericardial white adipose tissue stem cells induces a partial beige-like phenotype. PLoS One 11: e0157644. [Crossref]

21. Bargut TCL, Souza-Mello V, Aguila MB, Mandarim-de-Lacerda CA (2017) Browning of white adipose tissue: lessons from experimental models. Horm Mol Biol Clin Investig 31. [Crossref]

Copyright: (C2018 Guosheng LI. This is an open-access article distributed under the terms of the Creative Commons Attribution License, which permits unrestricted use, distribution, and reproduction in any medium, provided the original author and source are credited. 